\title{
REI :
}

L'iconomie : un modèle de l'économie numérique

\section{Pierre-Olivier Beffy, Jean-Marc Béguin, Pierre-Jean Benghozi, Laurent Bloch, Hugues Chevalier, Vincent Lorphelin, Michel Volle ${ }^{1 *}$}

Résumé : Le numérique automatise les tâches répétitives physiques et mentales. Le flux de travail que demande la production est donc faible en regard du \stock de travail qui la prépare. Le coût de production se réduit pratiquement au coût du capital fixe initial : le rendement d'échelle est croissant.

Il en résulte une cascade de conséquences dans la nature des produits, le régime des marchés, la stratégie des nations, l'organisation des entreprises, la nature du travail, la sociologie des pouvoirs et jusqu'à la psychologie des personnes.

L'économie présente connaît une crise de transition due à l'inadéquation du comportement des agents économiques (entreprises, consommateurs, État) en regard des ressources et des dangers qu'apporte le numérique. Pour sortir de cette crise il faut avoir une conscience claire de ces ressources et de ces dangers.

Nous proposons à cette fin le modèle, nommé iconomie, d'une économie numérique par hypothèse efficace.

Mots clés : numérique, automatisation, crise, iconomie.

Classification JEL : D43, L13, O14.

Le mot « informatique » allie $\mathrm{l}^{\prime}$ « automate » à $\mathrm{l}^{\prime}$ « information », cette dernière procurant à qui sait l'interpréter la forme intérieure qui lui permet d'agir ${ }^{2}$. Le mot «informatisation » désigne la dynamique du déploiement de l'informatique et de ses conséquences dans une entreprise, une institution ou un pays. À ces deux mots l'usage a substitué « numérique » au risque d'un appauvrissement que nous éviterons en lui donnant pour contenu celui des mots qu'il a supplantés.

Le numérique fait l'objet de jugements opposés. Nicholas Carr estime qu'il n'a aucune importance (Carr [7], 2003) car comme les entreprises en tirent toutes également parti il n'aurait aucune incidence sur leur compétitivité. Robert Gordon, héritier du paradoxe de Solow ${ }^{3}$, pense qu'il ne procurera jamais un gain de productivité comparable à celui qu'ont apporté la mécanisation et la maîtrise de l'énergie (Gordon [18], 2012).

D'autres auteurs ne partagent pas ce pessimisme. Au MIT Erik Brynjolfsson soutient que le numérique transforme en profondeur l'économie et dit que si ses effets sur la productivité semblent faibles, c'est en partie parce que les instruments de mesure sont devenus inadéquats, en partie parce

1 Les auteurs sont membres de l'Institut de l'iconomie, Paris.

2 Nous prenons ici «information » au sens de Simondon et non de Shannon : «L'information n'est pas une chose, mais l'opération d'une chose arrivant dans un système et y produisant une transformation. L'information ne peut pas se définir en dehors de cet acte d'incidence transformatrice et de l'opération de réception » (Simondon [43], 2010).

3 «You can see the computer age everywhere but in the productivity statistics » (Robert Solow, New York Review of Books, 12 juillet 1987). 
que l'économie est en transition et que les institutions ne se sont pas encore adaptées au numérique (Brynjolfsson et McAfee [31], 2011).

En France des chercheurs encouragés par le $\mathrm{CIGREF}^{4}$ ont produit une série d'études sur les effets du numérique dans les entreprises (Bounfour [5], 2016) et l'école de l'économie de la régulation voit dans le numérique un facteur déterminant de l'évolution des institutions (Boyer [6], 2018).

Les techniques du numérique ${ }^{5}$ font l'objet d'une abondante littérature destinée à des spécialistes. La plume des essayistes a été tentée par certaines d'entre elles, notamment par l'« intelligence artificielle » qui attise dans le public des espoirs et des craintes également extrêmes.

Nous avons nommé iconomie ${ }^{6}$ le modèle d'une économie numérique par hypothèse efficace (Saint Étienne [38], 2013 ; Volle [50], 2014 ; Rochet et Volle [46], 2015). Ce modèle n'est ni une représentation de l'économie présente, ni une prévision de l'économie future : c'est un repère placé à l'horizon du temps. Comme tout modèle il est schématique et ce schéma est fait pour orienter les intentions vers l'action judicieuse.

* * *

L'économie moderne s'est déployée à partir de la fin du XVIIIe siècle en s'appuyant sur la synergie de la mécanique et de la chimie auxquelles s'est ajoutée, vers la fin du XIXe siècle, l'utilisation du pétrole et de l'électricité (Gille [17], 1978). Elle a fait place à partir des années 1970 à l'économie numérique qui s'appuie sur la synergie de la microélectronique, du logiciel et de l'Internet. La mécanique, la chimie et l'énergie ne sont pas supprimées mais elles sont informatisées tout comme l'agriculture a été mécanisée et « chimisée » aux XIXe et XXe siècles, et leur part dans l'emploi se réduit.

Le numérique a ainsi provoqué la troisième « révolution industrielle ».

Pour étudier ses effets il faut s'appuyer sur ce que la théorie économique a plus de fondamental ${ }^{7}$ : (1) la distribution initiale des ressources entre les acteurs, (2) la fonction de production des entreprises, (3) la fonction d'utilité des consommateurs.

La spécification des ressources initiales, de la fonction de production et des préférences des consommateurs permet de poser un diagnostic sur une économie particulière. Or une révolution industrielle transforme à la fois les ressources initiales, la fonction de production des entreprises et jusqu'aux préférences des consommateurs, car ils réagissent à l'offre dont ils ont connaissance.

On ne peut donc conserver tels quels dans l'économie numérique ni le diagnostic que les économistes ont porté sur l'économie moderne, ni les prescriptions qu'ils ont formulées en s'appuyant sur le modèle néo-classique de l'équilibre général (Walras [51], 1883).

Ce texte a été élaboré par des économistes et praticiens de haut niveau appartenant à diverses disciplines (informatique, sociologie, ingénierie, etc.) et qui coopèrent au sein de l'Institut de l'iconomie.

4 Club Informatique des Grandes Entreprises Françaises.

5 Langages de programmation, algorithmes, systèmes d'exploitation, systèmes d'information, sécurité informatique, etc.

6 Du grec eikon, image, et nomos, organisation.

7 La « boîte d'Edgeworth [12]» (1881) en donne une représentation partielle mais éclairante. 
Il a pour but d'engager un dialogue avec la communauté des économistes en partant du constat des limites que rencontrent les modèles économiques usuels pour rendre compte des phénomènes qui se manifestent autour du numérique.

Il s'agit moins d'un article académique au sens strict que d'une contribution visant à nourrir une analyse pertinente de la situation présente en explorant des pistes qui certes existent déjà dans l'histoire de la théorie économique, mais ne sont pas les plus fréquentées.

Il propose quelques idées fondamentales pour éclairer l'économie numérique :

- les tâches répétitives étant automatisées, le travail se concentre dans les tâches qui demandent discernement et initiative : la main d'œuvre fait place au cerveau d'œuvre dont l'emploi exige un commerce de la considération ;

- le rendement d'échelle étant croissant, le marché obéit au régime de la concurrence monopolistique ;

- chaque produit est un assemblage de biens et de services élaboré par un réseau de partenaires ;

- le client choisit la variété du produit qui lui convient selon le rapport utilité subjective/prix ;

- l'économie numérique est une économie du risque maximum, une économie de la qualité et une économie de la compétence.

\section{L'iconomie}

Pour explorer l'iconomie nous adopterons le point de vue selon lequel l'Entreprise est, parmi les institutions, celle qui assure l'interface entre les ressources naturelles et le bien-être matériel de la population $^{8}$.

Si l'on accepte de nommer «nature » l'ensemble des ressources et obstacles que rencontrent les intentions et les actions humaines, une évidence s'impose : chaque « révolution industrielle » transforme la nature car elle modifie ces ressources et ces obstacles.

Ainsi la première révolution industrielle, celle de la mécanisation, a aménagé la nature en y introduisant des tunnels, viaducs, chemins de fer et canaux ; la deuxième y a introduit de nouvelles sources d'énergie et des moteurs (électrique, à combustion interne, à réaction).

La troisième, celle de l'informatisation et du numérique, a elle aussi transformé la nature, et pour comprendre la situation économique qui en résulte il importe de voir clairement ce que cette transformation a de spécifique.

Dans une entreprise chaque agent est ou sera assisté par son « ordinateur », interface vers une ressource informatique composée de documents, logiciels, mémoires, processeurs, et dotée d'ubiquité grâce à l'Internet ${ }^{9}$.

Cette ubiquité a supprimé nombre des effets de la distance géographique, qui n'existe pas sur l'Internet et dont les effets sur le transport des biens ont été pratiquement annulés par le système de la logistique des containers, elle-même informatisée ${ }^{10}$.

8 Le bonheur est le bien-être mental.

9 Le téléphone filaire avait procuré au signal vocal une ubiquité restreinte car exigeant la proximité d'un appareil téléphonique. Le téléphone mobile lui a procuré l'ubiquité absolue, et lorsqu'il est devenu un ordinateur avec le smartphone cette ubiquité s'est étendue à la ressource informatique : le corps humain lui-même s'est alors trouvé informatisé, tout comme il a été naguère mécanisé grâce à la bicyclette et à l'automobile.

10 Pour les produits non pondéreux le coût du transport par container est en effet négligeable. Les produits pondéreux restent par contre soumis à une contrainte géographique. 
La relation des entreprises avec la nature a été par ailleurs transformée par l'automatisation des tâches répétitives : des robots s'activent dans les usines, les aviateurs sont assistés par un pilote automatique, les tâches intellectuelles sont assistées par des « intelligences artificielles » (moteurs de recherche, aides au diagnostic, traducteurs automatiques, assistants personnels, etc.). Bénéficiant de la puissance des processeurs, de la taille des mémoires et du débit des réseaux, cette automatisation permet des actions qui étaient impossibles avant l'informatisation.

\section{Le cerveau d'œuvre}

Les tâches répétitives étant automatisées, la main d'œuvre, qui constituait naguère l'essentiel de l'emploi est remplacée dans l'iconomie par le cerveau d'ouvre: les entreprises demandent à l'agent qui travaille devant son « ordinateur » de savoir user de discernement et faire preuve d'initiative.

Ce cerveau d'œuvre résulte de la symbiose de l'être humain et de l'ordinateur ${ }^{11}$. Pour qu'une entreprise puisse réussir cette symbiose le partage du travail entre l'être humain et l'ordinateur doit être défini selon ce que chacun sait faire mieux que l'autre : à l'ordinateur le travail répétitif, qu'il exécute avec rapidité et fiabilité ; à l'être humain les tâches qui exigent du discernement devant les cas particuliers, de l'initiative devant l'imprévu, du jugement, de la créativité, de l'empathie, etc.

L'entreprise numérique délègue ainsi des responsabilités aux concepteurs, qui font face à la nature physique et aux besoins qu'il s'agit d'anticiper, ainsi qu'aux personnes de la première ligne qui produisent des services ${ }^{12}$ face aux clients.

Il n'est plus possible aujourd'hui d'organiser et gérer le travail comme on l'a fait dans l'économie moderne (Denis [10], 2014) car le cerveau humain ne peut être efficace que s'il se sait libre de penser, d'imaginer, de s'exprimer. Contrairement aux principes qui étaient ceux de l'organisation hiérarchique, l'entreprise doit donc déléguer au cerveau d'œuvre la légitimité (droit à la parole, droit à l'écoute, droit à l'erreur) qui correspond aux responsabilités dont elle le charge.

Alors que l'économie moderne a exploité des ressources naturelles physiques (charbon, pétrole, terres cultivables, air respirable, etc.) au risque d'accumuler une « dette écologique » (Jancovici et Grandjean [19], 2007), l'économie numérique met en exploitation la ressource naturelle mentale qui réside dans le cerveau humain et que l'organisation de la main d'œuvre avait laissée en jachère.

Il s'agit bien d'une ressource naturelle puisque la nature la crée lors de la naissance de chaque enfant, et contrairement aux ressources physiques dont le stock est limité cette ressource mentale est renouvelée à chaque génération : elle est donc inépuisable ${ }^{13}$.

Tandis que le cerveau d'œuvre travaille en symbiose avec la ressource informatique grâce à l'interface que lui offre un système d'information, l'organisation de l'entreprise s'appuie sur la synergie des actions individuelles.

Dans l'économie moderne cette synergie a pu résulter de la discipline qu'imposait la hiérarchie ${ }^{14}$, mais cette dernière ne peut plus avoir dans l'économie numérique le monopole de la réflexion, de la décision et de la parole : la synergie des compétences, condition nécessaire de la cohérence des

11 « The hope is that, in not too many years, human brains and computing machines will be coupled together very tightly, and that the resulting partnership will think as no human brain has ever thought and process data in a way not approached by the information-handling machines we know today » (Licklider [28], 1960).

12 Un service est « la mise à disposition temporaire d'un bien ou d'une compétence » (Demotes-Mainard [9], 2003).

13 Cela n'est pas contradictoire avec le fait qu'un individu puisse être parfois épuisé.

14 L'étymologie de « hiérarchie » est « pouvoir sacré », arché et hieros : l'organisation hiérarchique a sacralisé la fonction de commandement. 
actions et de leur efficacité collective, s'obtient d'une part par la cohésion du système d'information, d'autre part et surtout par l'adhésion à une orientation commune, facilitée par un « story telling » qui suscite l'adhésion des agents à des valeurs partagées. 


\section{Le régime du marché}

Les tâches répétitives étant automatisées, l'essentiel du coût de production se condense dans l'investissement initial en conception et ingénierie : c'est évident pour les logiciels et les circuits intégrés, et cela se retrouve dans les autres biens à proportion de leur informatisation.

C'est vrai aussi pour les réseaux (télécoms, transport, énergie, routes), dont la capacité dépend d'un dimensionnement initial, ainsi que pour les services, dont la production s'appuie sur un réseau de compétences.

Il en résulte que dans l'économie le coût marginal de la production est négligeable en regard du coût fixe initial. Le rendement d'échelle est donc croissant.

Ce fait renverse l'une des hypothèses sur lesquelles s'appuie le modèle de l'équilibre général, ainsi que les prescriptions que l'on en déduit : lorsque le rendement d'échelle est croissant, le régime du marché ne peut pas être en effet celui de la concurrence parfaite et en particulier la tarification au coût marginal est impraticable.

Le régime du marché sera soit celui du monopole naturel, qui concentre toute la production du produit en une seule entreprise, soit celui de la concurrence monopolistique ${ }^{15}$ sous lequel les entreprises échappent à la domination d'un monopole en produisant des variétés que leurs attributs qualitatifs distinguent les unes des autres.

Les produits qui comme le lingot de cuivre pur ne se prêtent à aucune différenciation qualitative étant en fait rares, la plupart des secteurs obéiront au régime de la concurrence monopolistique. Le modèle qui rend compte de cette situation n'a pas les mêmes qualités esthétiques que celui de l'équilibre général : contrairement à l'équilibre de la concurrence parfaite, par exemple, celui de la concurrence monopolistique ne maximise pas le surplus collectif.

La perte en «beauté » est cependant compensée par un gain en pertinence : le modèle de la concurrence monopolistique éclaire utilement des dimensions de la situation présente dont le modèle de l'équilibre général est incapable de rendre compte.

La concurrence monopolistique s'établira dans les secteurs de l'économie numérique dont le produit est susceptible d'une différentiation qualitative, c'est-à-dire en fait dans la plupart des secteurs : tandis que la concurrence parfaite a été le régime de référence de l'économie moderne, la concurrence monopolistique est le régime de référence de l'économie numérique.

La stratégie de l'entreprise est alors de différencier son produit, d'innover pour en produire une variété qui le distingue des autres afin de conquérir un monopole sur un segment du marché mondial. Ce monopole sera temporaire car les concurrents sauront imiter cette variété du produit. Considérons le marché du smartphone : Apple conquiert en 2007 le monopole de l'interface à écran tactile avec l'iPhone (Isaacson [23], 2011), mais cette interface est adoptée par tous les autres smartphones (Samsung, Huawei, etc.) à partir du milieu des années 2010. Le marché se découpe alors en segments selon les préférences des clients.

La lutte pour le monopole temporaire provoque un flux d'innovations. Le rôle du régulateur n'est plus alors de promouvoir la concurrence parfaite au risque d'entraver l'innovation, mais de régler la

15 Voir «Introduction à la concurrence monopolistique »,http://michelvolle.blogspot.com/2014/12/introduction-laconcurrence.html. 
durée du monopole temporaire qui doit être assez longue pour rentabiliser l'innovation et assez courte pour que le monopole ne s'endorme pas sur ses lauriers.

La dynamique de l'innovation apporte une croissance qualitative, progrès de l'adéquation des variétés du produit aux besoins des consommateurs ou utilisateurs. Cette adéquation sera mutatis mutandis d'autant plus exacte que le nombre des variétés est plus élevé, c'est-à-dire que le coût fixe est plus bas pour chaque niveau de finition et que la sensibilité des clients à la qualité est plus forte.

La croissance qualitative n'a pas de limite car on ne peut pas assigner de limite a priori à des besoins qui s'expriment en termes de qualité. Elle s'appuie sur la ressource naturelle inépuisable qu'est le cerveau d'œuvre et qui apporte une néguentropie susceptible d'améliorer le rapport entre la société et la nature : la croissance qualitative n'est pas soumise à la corrélation qui a été observée dans l'économie moderne (Jancovici [24], 2013) entre la croissance quantitative que le PIB mesure et la croissance de la consommation d'énergie ${ }^{16}$.

\section{Une économie de la qualité}

Sous le régime de la concurrence monopolistique la différenciation du produit se fait selon deux dimensions : l'une « verticale », celle du niveau de gamme où les variétés se distinguent par leur degré de finition et donc par leur coût de production ; l'autre « horizontale », où elles se distinguent à finition et coût de production égal par leurs attributs qualitatifs. À l'équilibre le prix des variétés ayant le plus haut degré de finition est plus élevé que celui des autres ; le client choisira la variété qu'il préfère selon le rapport «utilité subjective/prix ».

Le caractère subjectif de l'utilité est le moteur de la différentiation : si tous les clients avaient les mêmes goûts, le produit ne se prêterait pas à la différentiation et une seule variété serait offerte ; si en outre le rendement d'échelle est croissant le marché obéit alors au régime du monopole naturel. Nous supposons que cette situation est rare.

L'utilité réside dans les « effets utiles » (Moati [34], 2011) que le produit procure au client. Le plaisir et le prestige social qu'apporte la possession d'un bien peuvent figurer parmi ces effets : dans l'économie moderne la consommation d'un bien impliquait le plus souvent son achat, sa possession, puis sa destruction par usure ou obsolescence, enfin sa mise au rebut sous forme de déchet.

L'économie numérique invite à d'autres usages. Le client doit être aidé pour trouver la variété qui lui convient, l'Internet des objets permet la supervision et la télémaintenance des biens, les dépannages doivent être rapides, le respect de l'environnement incite à organiser le recyclage des biens à la fin de leur cycle de vie : la possession d'un bien fait place à la location ou à la tarification à l'usage, le consommateur devient un utilisateur plus sensible au service que lui procure un bien qu'à sa possession. Le covoiturage se substitue à la propriété d'une voiture, la musique portative se développe aux dépens du disque, etc.

Chaque produit est l'assemblage d'un bien et de services (communication téléphonique, voyage en avion, location d'une voiture, d'un appartement, etc.) ou un pur service (consultation médicale, expertise d'un avocat, etc.). La part des services est grandissante : label AOC, échange gratuit, commande en ligne, livraison en magasin, «made in France », image de marque, traçabilité, etc.

La qualité du produit dépendra souvent moins de celle du bien que de celle des services qu'il comporte : la compétence et l'amabilité du vendeur, la clarté des informations, la rapidité des dépannages, etc., importent plus pour le succès d'un produit que les attributs physiques du bien.

16 L'évaluation écologique du numérique doit faire le bilan de ces phénomènes et de la consommation de ressources non renouvelables (énergie et terres rares) qu'exigent la production et l'utilisation des ordinateurs. 
La différenciation du produit portera parfois sur les seuls services : deux variétés comportent alors le même bien, accompagné de services qui diffèrent selon le segment de clientèle.

L'entreprise qui ambitionne de conquérir un monopole sur un segment de clientèle doit avoir identifié ses besoins afin de définir le bien et les services qui composent le produit ainsi que le fonctionnement de leur assemblage : la concurrence monopolistique implique un marketing scientifique, différent du marketing commercial de l'économie moderne.

La théorie de l'utilité (Bentham[3], 1780) a été supplantée par la théorie de la valeur-travail (Ricardo [36], 1817) puis par la théorie néo-classique de la valeur de marché (Walras [51], 1883). Les métriques que produit l'économie numérique (Lorphelin, Saint-Étienne et Volle [47], 2015) remettent l'utilité à la première place.

Amazon mesure la qualité des contributions grâce à la question : « ce commentaire vous a-t-il été utile? ». Les clics des internautes alimentent des algorithmes qui permettent à Google de définir le prix des publicités, à TripAdvisor et à BlaBlaCar de classer hôtels et conducteurs.

Ces métriques aident à personnaliser l'offre : Netflix propose une liste de films à chaque téléspectateur, Vente-privée élabore des recommandations individuelles grâce au Big Data, les jeux sur smartphone s'adaptent à chaque joueur, l'assureur Aviva calcule ses primes en évaluant la conduite automobile grâce à une « applet » installée sur le smartphone de l'assuré.

$L^{\prime}$ « utilité ajoutée » est valorisée : des modèles « freemium », dont le prix est proportionnel au service rendu, se sont imposés en ligne. Les patients américains notent leurs médecins sur doctor.com, les élèves de HEC notent leurs professeurs. Le magazine Forbes rémunère ses journalistes en fonction de la fidélité de leurs lecteurs.

Ces métriques rendent mesurable toute action économique, même infime comme un clic ou relative à une contribution non marchande. Le champ de l'observation économique, limité auparavant à la sphère marchande, s'élargit ainsi jusqu'aux frontières du bien-être matériel. Les problèmes théoriques que pose le caractère subjectif de l'utilité sont résolus par la pratique : le prix d'un même produit, par exemple celui des billets d'avion, peut varier entre deux clients et entre deux instants avec la segmentation tarifaire (yield management). La répartition équitable des revenus entre les coproducteurs devient possible.

Les métriques révèlent de nouvelles ressources, transformées en autant de richesses dans la phase ascendante de la transformation numérique. La création de valeur contributive que préfigure Wikipédia pour les savoirs s'étend à l'innovation et aux décisions collectives. La valeur coopérative des maquettes 3D partagées lors de la conception des Airbus s'étend à la production et à la maintenance. La valeur relationnelle que révèlent les étoiles d'Uber ou de BlaBlaCar, qui notent les chauffeurs et les passagers, s'étend au leadership et aux services à la personne.

Des initiatives publiques vont dans le même sens : des associations comptabilisent le bénévolat pour rendre compte de leur utilité sociale ; France Stratégie propose, après la commission Stiglitz (Stiglitz, Sen et Fitoussi [14], 2009), des indicateurs de «croissance soutenable »; l'OCDE définit des indicateurs de bien-être ; l'union européenne développe des critères d'utilité environnementale et sociale ; la commission Sénard-Notat propose d'introduire dans le Code Civil la « raison d'être de l'entreprise » (Notat et Sénard [44], 2018). 
Les mesures du travail et du capital ont structuré la politique pendant deux siècles. La mesure de l'utilité, qui révèle la compétence relationnelle, la dynamique collective et le rôle de l'utilisateur, transforme le modèle économique et conduit à redéfinir la mission de l'entreprise.

L'entreprise de l'iconomie conçoit une multitude de modèles d'affaire divers ${ }^{17}$ pour accroître les richesses marchandes et non marchandes et orienter les externalités. L'exploitation de richesses non marchandes sobres en ressources énergétiques d'origine fossile favorise une croissance endogène et qualitative.

\section{Une économie de la compétence}

L'essentiel des emplois de l'iconomie se trouve d'une part dans la conception des produits et l'ingénierie de la production, d'autre part dans les services : conseil avant-vente, financement d'un prêt, information, maintenance, dépannage, remplacement et recyclage en fin de durée de vie, etc. Il en résulte que l'entreprise de l'iconomie possède des attributs qui étaient restés latents dans l'entreprise moderne : elle est psychosociologique et extravertie.

Extravertie, parce que la plupart des agents travaillent en relation avec le monde extérieur, alors que dans l'entreprise moderne ils étaient confrontés au formalisme interne de l'organisation : les services sont en relation avec les clients, la conception anticipe leurs besoins tout en devant obéir aux contraintes de la physique et de la logique.

Psychosociologique, parce que la répartition des responsabilités et de la légitimité, le rôle de la fonction de commandement, enfin l'image que chacun se fait de soi, de sa place dans la société et de son destin, sont là encore essentiellement différentes de ce qu'elles étaient dans l'économie moderne qui exigeait de la plupart des agents une obéissance mécanique.

Le contenu de l'action productive et son insertion dans le temps sont eux aussi transformés. Dans l'économie moderne chaque produit résultait (1) d'un travail de conception qui fournissait un prototype et une ingénierie de la production; (2) du travail de la main d'œuvre, qui reproduisait le prototype en de nombreux exemplaires. Le volume du travail de conception était négligeable en regard du volume du travail de la main d'œuvre.

Le choix stratégiques essentiels d'une entreprise (techniques, nature du produit, marché visé) étaient certes faits par un entrepreneur, mais la théorie économique l'ignorait ${ }^{18}$ car elle postulait que les idées judicieuses naissent de la connaissance des volumes et des prix relatifs ${ }^{19}$. La dialectique « capital/travail » était alors celle du « travail à effet différé » consacré à la fabrication des machines et du «travail à effet immédiat » qui les mettait en œuvre dans la production.

Dans l'iconomie la situation est différente. Si le produit est un logiciel, un circuit intégré ou toute autre chose dont la production est automatisée, le prototype sera reproduit à l'identique sans que cela ne nécessite pratiquement aucun travail : le produit est l'expression immédiate de l'intelligence de ses concepteurs, sans intervention d'une « main d'œuvre ».

Ce n'est donc pas du travail qui est stocké dans le capital mais de la conception, autrement dit de l'intelligence. Les logiciels et les ordinateurs que l'entreprise utilise pour automatiser la production sont des produits de l'« intelligence à effet différé » de ses concepteurs. Ils libèrent le cerveau

17 Benghozi, 2015.

18 L'œuvre de Schumpeter ( [40], 1947) est une exception.

19 Des Polonais avaient inventé l'engrenage à chevrons. Lorsque André Citroën le voit en 1900 il conçoit les améliorations que cela peut apporter à la mécanique : cette intuition physique sort du périmètre de la science économique de son époque. 
d'œuvre des tâches répétitives et lui permettent de se consacrer au travail intelligent « à effet immédiat » qui complète l'action de l'automate en répondant aux imprévus et à des détails auxquels les concepteurs de l'automate n'ont pas pu penser.

Alors que dans l'économie moderne le travail de la main d'œuvre était mesuré selon sa durée et le nombre des agents, le travail « intelligent » se mesure selon le niveau de compétence qu'il manifeste : la décision judicieuse, le diagnostic exact, se condensent sans délai dans l'esprit de l'agent compétent. Le temps de «travail » du cerveau d'œuvre est donc consacré à l'accumulation d'une compétence dont la manifestation, étant la réalisation d'un potentiel, est aussi rapide qu'une décharge électrique : l'action productive du cerveau d'œuvre est comme une succession d'éclairs.

La compétence du cerveau d'œuvre est un capital personnel que chaque agent accumule et entretient. Cette composante du capital de l'entreprise est potentiellement nomade : le management doit savoir la retenir.

La dialectique « capital/travail », ou pour mieux dire «stock/flux », prend dans l'iconomie une forme différente de celle qu'elle avait dans l'économie moderne.

Ce que l'on appelle « intelligence artificielle » n'est rien d'autre que le capital d'intelligence à effet différé des programmeurs et des concepteurs de la microélectronique, stocké dans des logiciels et des équipements informatiques. Ses effets sont inédits et donc surprenants : il étend le domaine du possible et transforme les ressources et obstacles auxquels les intentions humaines sont confrontées, donc la nature elle-même ; il commande, avec des mots écrits dans un programme, à des choses munies de masse et de volume, et semble accomplir les promesses ancestrales de la magie.

Il y a de quoi troubler les esprits, les enthousiasmer, les tourmenter, les inciter enfin à divaguer dans le monde imaginaire que suggèrent des expressions comme « intelligence artificielle », "réseau neuronal », « apprentissage profond », etc.

Résumons : la dialectique « capital/travail », ou « stock de travail à effet différé/flux de travail à effet immédiat », moteur de l'action productive, prend dans l'iconomie une forme dans laquelle le « travail » est remplacé par l'« intelligence » sous les deux formes d'un stock (de conception ou de compétence) à effet différé et d'un flux à effet immédiat. Derrière cette évolution se trouve enfoui un changement du rapport entre la pensée et la nature.

La pensée qui se manifeste dans un produit mécanique s'appuyait sur une modélisation des phénomènes naturels qu'elle mettait en œuvre : la civilisation de la mécanique a vu dans la science un capital de résultats jugés assez robustes pour fournir son socle à la vie en société.

La civilisation du numérique voit par contre dans la science la démarche qui bâtit les concepts nécessaires à l'action dans une situation particulière. Or pour bâtir des concepts il faut passer par une étape pré-conceptuelle, celle où l'intuition lance des explorations dans l'infini de l'inconnu.

Chacun ne peut certes agir à un instant donné qu'en fonction de la « grille conceptuelle » qui lui permet de discerner les objets de l'action, et des «théories » qui lui permettent d'anticiper les effets de son action. Mais chacun peut aussi être conscient de l'existence des choses que sa grille conceptuelle ne lui permet pas de voir et attentif aux faits qui peuvent contredire ses théories.

Le cerveau d'œuvre, qu'il soit occupé à la conception de nouveaux produits, à l'ingénierie de la production ou à la relation de service avec les clients, travaille sur la membrane qui sépare le « petit 
monde » organisé intérieur à l'entreprise du « grand monde » qui lui est extérieur. Comme il y rencontre des imprévus, des cas particuliers et des opportunités nouvelles ${ }^{20}$, il ne peut faire œuvre utile que s'il est ouvert au « grand monde » qui entoure la finitude du connu.

Dans l'entreprise moderne seuls des ingénieurs étaient invités à vivre dans ce « grand monde » Dans l'iconomie cela s'étend au cerveau d'œuvre, qui se trouve ainsi invité à agir en animateur conscient des effets que l'entreprise peut avoir sur le monde extérieur.

L'iconomie peut-elle connaître le plein emploi ? Certains estiment que l'automatisation va entraîner un chômage massif (McKinsey [32], 2017), d'autres disent que l'économie saura finalement employer toute la force de travail de la population comme après les deux révolutions industrielles précédentes.

Le fait est que le secteur tertiaire (Debonneuil [8], 2007) emploie d'ores et déjà trois quarts de la population active et on constate que le taux de chômage est faible dans les pays où la production est fortement robotisée. Ces deux phénomènes traduisent la profondeur du changement que le numérique apporte dans la définition des emplois et la nature des tâches à accomplir.

Le plus vraisemblable est que l'emploi évoluera comme il l'a fait à la suite de chacune des révolutions industrielles : après une pénible période de transition, le système éducatif fournira à l'économie les compétences dont elle a besoin et le plein emploi se rétablira, mais avec un contenu radicalement nouveau.

\section{Une économie hyper-capitalistique}

Quand le coût de production se concentre dans un coût fixe l'essentiel du travail est consacré à la formation d'un capital : le capital («travail mort » accumulé dans le capital fixe, « capital de compétence » des agents) devient le seul facteur de production. L'économie numérique est donc hyper-capitalistique et son développement nécessite une forte augmentation du capital disponible (Beffy [1], 2017).

Comme lors de chacune des grandes transitions technologiques l'accès au financement est une nécessité vitale. La disponibilité du financement permet de réaliser des projets rentables mais provoque aussi un déversement de l'épargne dans des projets non rentables, voire dans le financement de la consommation.

La difficulté d'anticiper le succès des nouveaux projets conduit dans un premier temps à une bulle d'endettement, l'économie numérique n'y a pas échappé dans les années 1990-2000. Ces bulles sont amplifiées par l'illusion d'une croissance forte et immédiate due non à l'innovation mais à la généralisation de l'endettement.

Or une évolution technologique ne peut accroître la productivité de l'économie qu'après un délai. Les signes les plus visibles et les plus immédiats de la transition se trouvent dans de nouveaux produits mais l'absorption de l'innovation n'est profonde que lorsqu'elle affecte également l'organisation de la production, la nature du travail et jusqu'aux habitudes des consommateurs. L'explosion de la bulle d'endettement retarde cette absorption car elle contraint la demande.

20 Que l'on pense à l'expérience que font les chefs de projet et les agents de la «première ligne ». 
Le cycle générationnel s'imbriquant avec le cycle technologique (Strauss et Howe [22], 1997), l'émergence de l'iconomie rencontre des obstacles psychosociologiques : l'économie numérique n'est pas perçue de la même façon par les générations anciennes, dont les habitudes de consommation et de travail sont bien ancrées, et par celles qui grandissent dans un environnement partiellement informatisé.

L'économie numérique favorise dans sa première phase une concentration de richesse au sein de la population et parmi les entreprises : des bulles financières la concentrent entre les mains des détenteurs du capital, les nouvelles entreprises numériques dégagent des rendements très supérieurs à ceux de l'économie moderne. L'accès au travail se déforme au profit des individus qui possèdent la compétence nécessaire au cerveau d'œuvre.

L'économie numérique est l'économie du risque maximum parce que l'essentiel du coût de production est dépensé avant que le produit ne soit mis sur le marché. Elle est exposée à un risque de prédation car rien n'est plus rentable pour un prédateur que de s'emparer d'un capital mal protégé. La puissance et la discrétion de l'informatique facilitent par ailleurs l'abus de biens sociaux, l'évasion fiscale, la corruption et le blanchiment (Robert et Backes [13], 2001 ; Saviano [39], 2006).

Le numérique a permis le développement de techniques financières dont la régulation n'a pas été anticipée : il a incité la Banque à « produire de l'argent » selon un jeu à somme nulle avec notamment les subprimes (Lewis [26], 2011) et le trading de haute fréquence qui encourage un « délit d'initié systémique » (Lewis [27], 2015 ; Gayraud [16], 2011). Le développement des cryptomonnaies se fait sans véritable contrôle faute d'une maîtrise des enjeux de ce type d'innovation financière.

Le glissement de la Banque vers la prédation est attesté par le montant des amendes que des banques ont accepté de payer pour éviter la publicité et le risque d'un procès ${ }^{21}$. Un comportement prédateur s'est répandu : des rémunérations annuelles qui dépassent une dizaine de millions d'euros représentent l'appropriation d'un patrimoine et non le salaire d'un travail ni la compensation d'un risque.

Le régulateur se trouve alors coincé entre la nécessité d'encadrer les pratiques économiques et le risque d'entraver l'émergence de l'iconomie. Tandis que la théorie économique s'est construite autour de l'hypothèse de l'échange équilibré et a supposé la prédation négligeable, elle doit considérer aujourd'hui la dialectique qui s'est instaurée entre l'échange équilibré et la prédation dans l'économie numérique (Volle [48], 2008) : outre le réglage de la durée des monopoles temporaires, le régulateur de l'iconomie a pour mission de contenir la prédation.

\section{Ingénierie et iconomie}

L'entreprise numérique conjugue une ingénierie proprement informatique, celle du « système d'information » et des automatismes, avec l'ingénierie physique qui s'applique aux matériaux que la production transforme (Meinadier [33], 2002). Il faut leur ajouter une « ingénierie d'affaires » car pour limiter les risques la production sera le fait d'un réseau de partenaires.

La synergie des cerveaux d'œuvre mobilise les couches de l'ingénierie du système d'information (Volle [49], 2010) : ingénierie sémantique, ingénierie des processus et du contrôle, ingénierie stratégique, ingénierie d'affaires.

21 D'après le Boston Consulting Group les cinquante plus grandes banques ont payé 345 milliards de dollars d'amendes entre 2009 et 2017. 
L'ingénierie de la matière est soumise aux contraintes de la physique tout en étant pénétrée par l'informatique : logiciels de simulation pour la conception des produits, automatisation et informatique embarquée pour leur fonctionnement.

La physique des choses s'entrelace ainsi, de façon intime, avec celle des processeurs, mémoires et réseaux, ainsi qu'avec la logique de la programmation. Pour concevoir un tel système l'ingénierie a recours à des « modèles en couches » qui articulent des logiques usant chacune d'un protocole qui lui est propre, et communiquant par des interfaces (Tanenbaum [45], 1987).

Outre les entreprises, l'ingénierie concerne les nations. La concurrence monopolistique s'exerce en effet aussi à leur niveau selon un jeu stratégique qui interdit la naïveté : dans la «guerre économique »en cours chaque nation doit savoir tirer parti du numérique pour conforter la qualité de ses produits sur le marché mondial et équilibrer sa balance des paiements. Il ne suffit pas de s'émerveiller devant les start-ups, ni de suivre à répétition la mode qui enfle une bulle médiatique autour d'une technique, puis d'une autre.

Les nations qui maîtrisent le mieux les techniques fondamentales du numérique seront les mieux placées pour en tirer parti, voire s'en réserver l'utilisation ${ }^{22}$.

L'indépendance de l'Europe nécessite une maîtrise de l'ensemble de cette filière (Morin-Desailly [35], 2013). L'importance de la maîtrise d'un secteur dépend cependant de sa part dans la valeur ajoutée de la filière : les secteurs les plus importants sont ceux des techniques fondamentales (microélectronique, logiciel système et réseau) tandis que les secteurs proches de l'utilisation (ordinateurs, logiciels d'application) représentent une part relativement modeste de la valeur ajoutée.

- terres rares : la Chine dispose d'un monopole mais on peut espérer une action correctrice ; - optique et photolithographie : l'Europe dispose de leaders mondiaux avec Zeiss et ASML et d'entreprises de taille moyenne dans le bassin industriel de Grenoble ;

- microélectronique : l'entreprise franco-italienne STMicroelectronics est un des six acteurs mondiaux ; elle est accompagnée du néerlandais NXP, de l'allemand Siemens et d'entreprises de taille moyenne ; l'usine Global Foundries de Dresde appartient au fonds souverain émirati ATIC. Le secteur est menacé par les progrès chinois : la Chine ne dispose pas encore de certaines technologies essentielles mais elle les possédera dans quelques années ;

- fabrication d'ordinateurs : l'essentiel de la production sur le sol européen est le fait d'acteurs non européens comme Dell ;

- logiciel système et réseau : les positions européennes se limitent à la contribution à des projets de logiciel libre : cette absence laisse la domination de ce secteur à Google, Microsoft, Apple et Linux ;

- Cloud et Data Centers : le français OVH est un des leaders mondiaux, mais l'essor des acteurs du Cloud Computing (Amazon, Microsoft, Google) risque de le mettre en difficulté ; la tentative des pouvoirs publics pour créer un «Cloud souverain » est allée à l'échec ;

- logiciel d'application: seuls l'allemand SAP et le français Dassault Systems sont des leaders mondiaux mais ce secteur, très évolutif, reste ouvert à l'innovation.

Pour assurer son indépendance dans l'économie numérique la France devra restaurer le potentiel qui a été dilapidé dans les décennies récentes (Bull, Alcatel, France Télécom...) et se rapprocher de ses partenaires européens. Les politiques publiques sont attirées par les secteurs de basse intensité capitalistique comme l'« intelligence artificielle », mais un pays ne peut pas être une puissance industrielle sans occuper une position forte dans les secteurs clés.

22 La réglementation ITAR permet aux États-Unis de bloquer la vente de certains composants électroniques à usage militaire et spatial. 
La France avait un industriel dans le créneau des composants électroniques à usages militaires et spatiaux, Altis Microelectronics, mais il a été acheté par une entreprise germano-belge qui quitte ce créneau. Cette lacune est un risque majeur pour l'industrie européenne.

Le développement de l'Internet des objets (IoT) ouvre des marchés gigantesques sur lesquels il reste des places à prendre, par exemple dans les matériels de transport (ferroviaire, automobile, aéronautique).

\section{Éducation et iconomie}

La première révolution industrielle a demandé l'alphabétisation généralisée, après la seconde il a fallu former des ingénieurs, techniciens et ouvriers qualifiés. L'informatisation du monde qui a commencé il y a un demi-siècle suppose que l'ensemble de la population acquière une compréhension de la science informatique.

L'enseignement de l'informatique ne peut aucunement consister en une formation à ses usages, il doit s'appuyer sur son socle scientifique qui s'articule autour de quatre concepts fondamentaux : information, programme, langage, machine (Dowek [11], 2011 ; Berry [4], 2017).

De même que l'on ne peut pas comprendre les mathématiques sans avoir résolu quelques équations et démontré quelques théorèmes, on ne peut pas comprendre l'informatique si l'on n'a pas écrit quelques programmes et installé quelques systèmes d'exploitation.

Les dirigeants de l'économie et de la politique, dont la fonction n'est pas d'être des experts, ne pourront prendre des décisions raisonnables que s'ils ont acquis une intuition exacte de l'informatisation, c'est-à-dire une culture de l'informatique. Or ce résultat ne pourra être atteint que si cette culture est présente, comme dit Gilbert Simondon, dans la «grande masse » de la population $^{23}$.

Il faut donc que chacun puisse au cours de sa scolarité se familiariser avec cette science, comme depuis des décennies chacun s'est familiarisé avec les problèmes que les ingénieurs cherchent à résoudre avec les mathématiques et la physique. Cela suppose d'introduire dans l'enseignement secondaire un cours d'informatique d'un volume équivalent à celui du cours de mathématiques : il est possible de s'inspirer du Royaume-Uni ou d'Israël (Gal-Ezer et Harel [15], 2010) qui ont accompli cette réforme. Il faudra donc créer un corps professoral dont la compétence sera attestée par le master d'informatique, suivi par le CAPES ou l'agrégation.

\section{S'orienter vers l'iconomie}

Les dirigeants des entreprises et de l'État, les économistes, les personnes qui influencent l'opinion ont-ils pris la mesure des changements que le numérique provoque ? Voient-ils clairement les possibilités qu'il apporte et les dangers qui les accompagnent ? Ont-ils défini l'orientation qui permettra de sortir de la crise actuelle ?

Des causes structurelles sont invoquées pour expliquer cette crise (niveau des salaires, des impôts, des taxes, déficit du budget de l'État, etc.) et il est proposé d'y remédier par des « réformes structurelles ».

23 «C'est la culture qui gouverne l'homme, même si cet homme gouverne d'autres hommes et des machines. Or, cette culture est élaborée par la grande masse de ceux qui sont gouvernés ; si bien que le pouvoir exercé par un homme ne vient pas de lui à proprement parler, mais se cristallise et se concrétise seulement en lui : il vient des hommes gouvernés et y retourne » (Simondon [42], 1958). 
L'expérience de la vie dans les entreprises suggère cependant une autre explication : si l'économie est en crise, c'est parce que la majorité d'entre elles ne se sont pas adaptées au numérique, qu'elles se sont mal informatisées.

Les apports du numérique sont en effet perçus de façon confuse. À l'enthousiasme d'un Michel Serres [41] (2012) font pendant les inquiétudes de ceux qui prétendent que « trop d'information tue l'information », que «l'automatisation tue l'emploi », et qui ne voient pas que le danger réside plutôt dans la violence endémique de l'économie du risque maximum, violence à laquelle le numérique procure des outils puissants.

Certains croient comme Jeremy Rifkin [37] (2013) que la révolution industrielle n'est pas due au numérique mais à la transition énergétique. D'autres (Latouche [25], 2010) appellent de leurs vœux une décroissance seule capable, pensent-ils, de limiter la prédation subie par la nature, car ils ne conçoivent pas la possibilité d'une croissance qualitative.

$* \quad *$

Dans l'attente d'une compréhension des conséquences du numérique le comportement des agents économiques - consommateurs, entreprises, État - est contraire aux conditions nécessaires de l'efficacité que le modèle de l'iconomie met en évidence. Depuis les années 1970 la doctrine néolibérale (Hayek [21], 1944) a orienté les stratégies au rebours de ce qu'il aurait fallu faire pour tirer parti du numérique :

- alors que l'État est l'«institution des institutions » (Hauriou [20], 1925) son rôle stratégique a été nié pour faire place à l'autorégulation des marchés ;

- la réglementation européenne, inspirée par la croyance en l'efficacité de la concurrence parfaite, a brisé la cohésion des infrastructures (télécoms, chemins de fer, électricité etc.) en répartissant leurs organes entre des entités qui se disputent le résultat.

La France a connu divers errements :

- alors que le numérique a transformé les conditions pratiques de la production, nombre de dirigeants considèrent l'informatique comme une discipline ancillaire et la traitent en conséquence ; - alors que dans l'iconomie la compétitivité se gagne par l'innovation en conquérant un monopole temporaire sur un segment des besoins, les entreprises ont misé sur la production de masse de produits standards et sur la concurrence par les prix ;

- alors que les consommateurs doivent se procurer des «effets utiles » (Moati [34], 2011) en choisissant selon le rapport subjectif qualité/prix, la distribution les a incités à rechercher toujours le prix le plus bas ;

- alors que les produits doivent être des assemblages de biens et de services, la production des biens a été délocalisée vers des pays à bas salaires, les services ont été négligés ;

- alors que la production doit être réalisée par un réseau de partenaires, la sous-traitance a été préférée au partenariat entre égaux ;

- alors que l'entreprise emploie non plus une main d'œuvre mais un cerveau d'œuvre, elle impose à celui-ci un rapport hiérarchique qui le stérilise ;

- alors que l'endettement de la nation résulte de l'accumulation du déficit de la balance des transactions courantes, l'attention s'est focalisée sur la dette de l'État.

Le système productif a été maltraité :

- les régulateurs, croyant que l'efficacité ne peut être atteinte que sous le régime de la concurrence parfaite, s'opposent aux stratégies que demande la concurrence monopolistique ;

- le secteur financier, mondialisé et automatisé, a été déchaîné par la dérégulation et incité à

« produire de l'argent » en parasitant le système productif ; 
- la délocalisation a pérennisé des techniques obsolètes et retardé l'automatisation ;

- la R\&D s'est le plus souvent limitée à la mise en évidence d'idées judicieuse, laissant à d'autres

pays le soin d'industrialiser les produits qui en tirent parti (Morand et Manceau [30], 2007) ;

- les services bancaires informatisés blanchissant efficacement les profits du crime organisé, celui-

ci s'efforce de contrôler le système productif et rivalise avec l'État pour instaurer un pouvoir politique de type féodal.

Ces comportements ont créé une crise durable et un désarroi dans l'opinion : alors que chacun estime avoir droit à la satisfaction de ses besoins et à un statut social, nombreux sont ceux qui se disent hostiles à la science, à la technique et aux entreprises qui les leur procurent.

Le réalisme exige de placer l'iconomie à l'horizon du futur pour s'orienter vers la sortie de la crise, la restauration du plein emploi et l'équilibre des échanges avec l'extérieur :

- l'économie étant désormais numérique, l'efficacité est conditionnée par la qualité de

l'informatisation des entreprises et des institutions ;

- comme l'emploi se condense dans le cerveau d'œuvre, l'entreprise doit renoncer à l'organisation hiérarchique pour instaurer un « commerce de la considération » dans les relations entre personnes et entre spécialités, ainsi qu'avec ses clients, fournisseurs et partenaires ; comme la fonction de production est à coût fixe, chaque produit doit être un assemblage de biens et de services, élaboré par un partenariat ;

- comme les marchés obéissent au régime de la concurrence monopolistique, la stratégie des entreprises doit être de conquérir un monopole temporaire sur un segment mondial des besoins ;

- l'État doit orienter les entreprises vers l'iconomie en (1) assurant la qualité de l'informatisation des grands systèmes de la nation (éducatif, sanitaire, judiciaire, fiscal, militaire, etc.), (2) régulant la concurrence monopolistique, (3) promulguant les lois qui permettent au système judiciaire de contenir la violence de l'économie du risque maximum et la prédation ;

- la science informatique doit recevoir dans l'enseignement secondaire une importance comparable à celle des mathématiques ;

- des infrastructures doivent être développées, des compétences formées, la créativité encouragée et le savoir-faire protégé.

L'innovation transformant continuellement le monde et faisant émerger une grande diversité de modèles d'affaire, la politique économique ne peut pas se borner aux prescriptions du libre échange des produits et des capitaux (List [29], 1841) : des positions doivent être prises et maintenues dans les techniques fondamentales.

L'État doit donc savoir défendre comme autant de forteresses certaines infrastructures, entreprises et recherches. Sa stratégie comme son action régulatrice doivent répondre aux risques que comporte l'économie numérique : le marché est global, la concurrence est mondiale et certaines entreprises dominantes acquièrent, comme l'ont fait les GAFA, une ampleur géopolitique. 


\section{Références}

[1] Pierre Olivier Beffy. Talkin’ 'bout 20 generations. Exane Paribas, 2017.

[2] Pierre-Jean Benghozi. Nouveaux modèles d'affaire ou modèles économiques ? In L'intelligence iconomique. De Boeck, 2015.

[3] Jeremy Bentham. An Introduction to the Principles of Morals and Legislation. 1780.

[4] Gérard Berry. L'hyperpuissance de l'informatique - Algorithmes, données, machines, réseaux. Odile Jacob, 2017.

[5] Ahmed Bounfour. Futurs numériques, transformation numérique. Eska, 2016.

[6] Robert Boyer. La théorie de la régulation au fil du temps. EMSHA, 2018.

[7] Nicholas Carr. IT does'nt matter. Harvard Business Review, mai 2003.

[8] Michèle Debonneuil. L'espoir économique : Vers la révolution du quaternaire. Bourin, 2007.

[9] Magali Demotes-Mainard. La connaissance statistique de l'immatériel. INSEE, 2003.

[10] Jean-Philippe Denis. Introduction au Hip-Hop Management. Éditions EMS, 2014.

[11] Gilles Dowek. Les quatre concepts de l'informatique. In Sciences et technologies de l'information et de la communication en milieu éducatif: Analyse de pratiques et enjeux didactiques. New Technologies Editions, 2011.

[12] Francis Edgeworth. Mathematical Psychics. Kegan Paul, 1881.

[13] Denis Robert et Ernest Backes. Révélation\$. Les Arènes, 2001.

[14] Joseph E. Stiglitz, Amartya Sen et Jean-Paul Fitoussi. Richesse des nations et bien-être des individus : performances économiques et progrès social. Odile Jacob, 2009.

[15] Judith Gal-Ezer and David Harel. Curriculum and course syllabi for a high-school program in computer science. 2010.

[16] Jean-François Gayraud. La grande fraude. Odile Jacob, 2011.

[17] Bertrand Gille. Histoire des techniques. Gallimard, Collection de la Pléiade, 1978.

[18] Robert Gordon. Is U.S. Economic Growth Over? Faltering innovation confronts the six headwinds. Center for Economic Policy Research, 2012.

[19] Jean-Marc Jancovici et Alain Granjean. Le plein s'il vous plaît! Points, 2007.

[20] Maurice Hauriou. Théorie des institutions et de la fondation. 1925.

[21] Friedrich Hayek. The Road to Serfdom. 1944.

[22] William Strauss et Neil Howe. The Fourth Turning. Broadway Books, 1997.

[23] Walter Isaacson. Steve Jobs. Simon \& Schuster, 2011.

[24] Jean-Marc Jancovici. Transition énergétique pour tous : ce que les politiques n'osent pas vous dire. Odile Jacob, 2013.

[25] Serge Latouche. Le pari de la décroissance. Fayard, 2010.

[26] Michael Lewis. The Big Short : Inside the Doomsday Machine. Penguin, 2011.

[27] Michael Lewis. Flash Boys - A Wall Street Revolt. W. W. Norton \& Co, 2015.

[28] Joseph Licklider. Man computer symbiosis. IRE Transactions on Human Factors in

Electronics, 1960.

[29] Friedrich List. Das nationale System der politischen Ökonomie. 1841.

[30] Pascal Morand et Delphine Manceau. Pour une nouvelle vision de l'innovation. La documentation française, 2007.

[31] Erik Brynjolfsson et Andrew McAfee. Race Against The Machine : How the Digital Revolution is Accelerating Innovation. Digital Frontier Press, 2011.

[32] McKinsey. Jobs Lost, Jobs Gained: Workforce transitions in a time of automation.

McKinsey\&Co, décembre 2017.

[33] Jean-Pierre Meinadier. Le métier d'intégration de système. Hermès, 2002.

[34] Philippe Moati. La nouvelle révolution commerciale. Odile Jacob, 2011.

[35] Catherine Morin-Desailly. L'union européenne, colonie du monde numérique ?, 2013.

[36] David Ricardo. On the Principles of Political Economy and Taxation. 1817. 
[37] Jeremy Rifkin. The Third Industrial Revolution. Palgrave Macmillan, 2013.

[38] Christian Saint-Étienne. L'iconomie. Odile Jacob, 2013.

[39] Roberto Saviano. Gomorra. Viaggio nell'impero economico e nel sogno di dominio della Camorra. Mondadori, 2006.

[40] Joseph Schumpeter. Capitalism, Socialism and Democracy. George Allen and Unwin Ltd, 1947.

[41] Michel Serres. Petite Poucette. Le Pommier, 2012.

[42] Gilbert Simondon. Du mode d'existence des objets techniques. Aubier, 1958.

[43] Gilbert Simondon. Communication et information. Éditions de la transparence, 2010.

[44] Nicole Notat et Jean-Dominique Sénard. L'entreprise, objet d'intérêt collectif. 2018.

[45] Andrew Tanenbaum. Operating Systems : Design and Implementation. Prentice Hall, 1987.

[46] Claude Rochet et Michel Volle. L'intelligence iconomique. De Boeck, 2015.

[47] Vincent Lorphelin, Christian Saint-Étienne et Michel Volle. Le triomphe de l'économie de l'utilité. Le Monde, 20 juin 2015.

[48] Michel Volle. Prédation et prédateurs. Economica, 2008.

[49] Michel Volle. Systèmes d'information. Encyclopédie des techniques de l'ingénieur, 2011.

[50] Michel Volle. iconomie. Economica, 2014.

[51] Léon Walras. Théorie mathématique de la richesse sociale. 1883. 